\title{
Smart board level machine vision system for online inspection : A Review
}

\author{
Namratha $M R^{1}$, Jyothi Pramal ${ }^{2}$, Praveen $\mathbf{J}^{3}$, Raghavendra Rao ${ }^{4}$ \\ M.Tech Student, Dept. of ECE, VLSI design and embedded systems, AIET, Mijar, Moodbidri, India ${ }^{1}$ \\ Assistant Professor, Dept. of ECE, AIET, Mijar, Moodbidri, Karnataka, India ${ }^{2}$ \\ Sr. Associate Professor, Dept. of ECE, AIET, Mijar, Moodbidri, Karnataka, India ${ }^{3,4}$
}

\begin{abstract}
A simple and low cost machine vision system for fault detection and identification is envisaged. The proposed system works on a Beagle Board single board computer. The system can be used to detect cracks or texture differences in classifying objects, especially in food processing industries. It essentially uses a camera for the image capture and process the image in the Beagleboneblack. Currently an online machine vision system on a conveyor line is made up of several cameras that are networked to a single computer. The camera provides the images at high rates and the software on the computer does the processing and makes the decisions to reject the product or not. The goal of this project is to design a smart board level machine vision system using a beagleboneblack with its own image sensors/cameras. The system will have its own algorithms for common machine vision tasks and will be networked to a main system to get output results.
\end{abstract}

Keywords: Machine vision system, Beagleboneblack, Image processing, Fault detection.

\section{INTRODUCTION}

\section{A. An Introduction to Digital Image Processing}

The Digital image processing technology has got great attention and considerable development in recent years. However the present image processing systems are not satisfactory with respect to real time applications and constraints. The traditional image processing techniques rely on large quantities of electronic computing devices, which results in enormous maintenance, transportation and other Expenditures. These systems are based on PC and are therefore too bulky to have portability; the desk-top Operating system does not have real-time character. Embedded platform is excellent due to its features of smaller size, low-cost, low power consumption and low maintenance. Therefore, developing an image processing system on the embedded platform can reduce the production and maintenance costs, improves the reliability and controllability. In this regard, this project proposes an image processing system based on embedded platform using very low cost BeagleBoneblack

\section{B. An Introduction to Embedded systems}

Embedded system is a special computer system which centers on application, Takes computer technology as basis and adapts to the requirements of application system for function, reliability, cost, volume and power consumption and whose software and hardware can be tailored. Embedded technology has a natural connection with real-time property: an embedded system is designed for a specific purpose and it is usually restricted by space, cost, storage and bandwidth, etc., so it must be made to measure on hardware and software to the utmost extent to improve efficiency and such results finally increase its real-time property.

C. An Introduction to quality inspection of rice

Rice is one of the most consumed foods in the world. With the improvement of standard of living of human being, higher quality of food is required than before. The quality of rice is based on variety of properties such as texture, color, size shape, number of broken rice kernel, stones mixed, yellow particle mixed with it etc. For on line measurement a transport system is used to position the rice kernel below the camera and for sorting a discharging device is used to collect the classified kernels in different containers. Machine Vision System is relative expensive, influenced by external light conditions and needs an experienced person to setup the system. In the conventional rice sorter, image of fallen rice from a shoot is captured, and then rice color-stone is compared with some threshold value. If the rice color stone is below the threshold value the rice is rejected as damaged rice. For this conventional rice sorter, when percentage of paddy in polished rice exceeds $1 \%$, it is difficult to exclude all paddies. Furthermore if rice flow exceeds a few thousands (kgh), the recognition percentage is below $90 \%$ and recognition ability is not always guaranteed. While small flow of rice guarantees recognition ability, this matter leads a low efficiency for rice sorting. So there is a need to develop a low cost and reliable system.

\section{An Introduction to OpenCV (Open Computer Vision)}

Originally an Intel research initiative, OpenCV is a crossplatform open source computer vision library, mostly employed for its real time image processing performance. It aims to provide well tested, optimized, open source implementations of, state of the art image processing and computer vision algorithms. OpenCV is an open source computer vision. The library is written in $\mathrm{C}$ and $\mathrm{C}++$ and runs under Linux, Windows and Mac OS $\mathrm{X}$. There is active development on interfaces for Python, Ruby, Matlab, and other languages. Multiple languages bindings are available for OpenCV such as OpenCV Python has 
been created for Python as well as the bindings automatically built with swig. OpenCV was designed for computational efficiency and with a strong focus on real time applications. OpenCV is written in Optimized $\mathrm{C}$ and can take advantage of multicore processors. One of the OpenCV's goal is to provide a simple-to-use computer vision infrastructure that helps people build fairly sophisticated vision applications quickly. The OpenCV library contains over 500 functions that span many areas in vision, including factory product inspection, medical imaging, security, user interface, camera calibration, stereo vision, and robotics.

\section{LITERATURE SURVEY}

Bharat K. Patel, R. C. Patel [2], presented their research work on An Efficient method for detecting impurity from Rice mixture using Digital Embedded System. In their work; they developed a faster, automatic, precise, economic and real time prototype system for detecting impurity from rice using ARM controller. The system uses huge value of the color impurity to detect them real time from the mixture of rice. With the help of OpenCV (Open computer vision) library an algorithm is developed to track impurity from an embedded device to track impurity from rice mixture in real time. It is useful in quality inspection of rice grain in agriculture industry.

These systems are based on PC and are therefore too bulky to have portability; the desk-top operating system does not have real time character. Embedded platform is excellent due to its features of smaller size, low-cost, low power consumption and low maintenance. Therefore, developing an image processing system on the embedded platform can reduce the production and maintenance costs, improves the reliability and controllability. And here they proposed an image processing system based on embedded platform using very low cost ARM controller Raspberry Pi board (700Mhz.).

Smita Shelke, Prof. A.P Phatale [3], presented their research on an automatic grading system based on machine vision their work is based on identifying the food on the basis of its color, shape size as well as the moisture content. This concept has been developed especially for the food items. Color spectrum technique has been used here a numerous sensors have been developed for analyzing the other parameters of the food item.

And the main objective of this system is used to determine the various parameters of an onion meant for the export, and segregate them taking into consideration the required elements /factors that would meet the qualitative norms/criteria that has been fixed as per the export rules set by the government. In their work they identify the food on the basis of its color, shape as well as size so we can use color spectrum technique. Color spectrum technique is an easy method to differentiate between various colors therefore we are going to design food separation system, which will separate different food particles based on their colors. And their work is based on the fundamentals of optics and digital electronics.
Harpreet Kaur, Baljit Singh [4], presented their research work on classification and grading of rice using multi class SVM. In their work; they proposed machine algorithm to grade (Premium, Grade A, Grade B and Grade C) the rice kernels using Multi-Class SVM. Maximum Variance method was applied to extract the rice kernels from background, then, after the chalk has been extracted from rice. The percentage of Head rice, broken rice and Brewers in rice samples were determined using ten geometric features.

Multi-Class SVM classified the rice kernel by examining the Shape, Chalkiness and Percentage of Broken (Head Rice, Broken and Brewers) kernels. The SVM classify accurately more than $86 \%$. Based on the results, it was concluded that the system was enough to use for classifying and grading the different varieties of rice grains based on their interior and exterior quality.

Chetna V. Maheshwari [5], presented their work on machine vision technology for oryza sativa 1.(rice). In their work; the basic problem of rice industry for quality assessment is defined which is traditionally done manually by human inspector. Their reviews various quality evaluation and grading techniques of Oryza Sativa L.(rice) in food industry. Machine vision provides one alternative for an automated, non-destructive and cost-effective technique.

Machine vision in food has broadened its range of applications from grains, cereals, fruits to vegetables including processed products in which there is a high degree of quality achieved as compared to human vision inspection. They quantify the qualities of various rice varieties and figure out features which directly or inversely affect the quality of the rice. Based on these features a generalized formula of quality is proposed to be used for quality evaluation of any type of rice variety.

Ms. Shubhada.K. Nagrale,Mr. S.T.Bagde [6], presented a paper on Application of Image Processing For Development of Automated Inspection System. In their work a model of automated inspection system is presented in this conceptual paper. Image processing is used for inspection of part. It is assumed that the part after going through many previous operations comes to inspection system where the weight of the part as well as geometry made on that part is detected and later decided whether it is to be accepted or rejected with the help of image processing technique. Using MATLAB software a program is developed and pattern or geometry is detected.

Chetna v. Maheshwari, Kavindra r. Jain and Chintan k. Modi [7], presented their work on non-destructive quality analysis of indian gujarat-17 oryza sativa ssp indica(rice) using image processing. In their work; they presented a solution for quality evaluation and grading of Rice industry using computer vision and image processing. In their work basic problem of rice industry for quality assessment is defined which is traditionally done manually by human inspector.

Machine vision provides one alternative for an automated, non-destructive and cost-effective technique. With the 
help of proposed method for solution of quality designed and fabricated based on machine vision system. assessment via computer vision, image analysis and This system was composed of conveyor belt, lighting box, processing there is a high degree of quality achieved as controlling and processing system unit and sorting unit. compared to human vision inspection. They proposed a Color feature is the most important parameter in new method for counting the number of Oryza sativa L classification and sorting of raisins. In order to carry out (rice seeds) with long seeds as well as small seeds using image processing and to extract useful features of captured image processing with a high degree of quality and then quantify the same for the rice seeds based on combined measurements.

Latha, J. and N. Devarajan [8], presented their research work on In-Process Vision Inspection Systems for Sorting using Image Processing Techniques. In their work they focused on the purpose of machine vision is the desire to provide real time machines with visual abilities. A real time system is developed and is interfaced with the mechanical structure to be used in automobile industry.

Dynamic thersholding is used and image pre-processing techniques are considered and implemented namely ellipse and circle algorithm. The developed machine vision system consists of a mechanical structure, DIO card for communication and pneumatic components. The proposed model is tested on real time for segregation and mix up of automobile nut samples.

Roya H assankhani, Hossein Navid [9], presented their research work on Potato Sorting Based on Size and Color in Machine Vision System. In their research 110 numbers of potatoes from Agria variety were prepared. Samples were pre-graded based on quantitative, qualitative and total factors manually before sorting. Quantitative, qualitative and total sorting in Machine vision system was performed by improving images quality and extracting the best thresholds. The accuracy of total sorting was $\% 96.823$.

Manish Chhabra, Abhishek Gupta [10], presented their research work on Automated Detection of Fully and Partially Roped Mango by Machine Vision. In their work; the quality of the mango can be judge by its length, thickness, width, area, etc. In this paper on the basis of simple mathematical calculations different parameters of a number of mangos are calculated. Their work focused on the classification of mangoes using morphological Operations. A video containing mangoes hanging from the trees is made and used as the input to this algorithm. The video is read frame by frame and the within one frame morphological operations, watershed algorithm and analysis and segmentation are applied.

The mango types used in this study were Ripe Mango, Unripe Mango. In this work the application of neural network is used for assessment of mango. The contours of ripe and unripe mangoes have been extracted, precisely normalized and then used as input data for the neural network. The network optimization has been carried out and then the results have been analyzed in the context of response values worked out by the output neurons.

Mahdi Abbasgolipour [11], presented their research work on Sorting Raisins by Machine Vision System. In their research, an apparatus for sorting raisins has been images by machine vision a highly efficient algorithm was developed and implemented in Visual Basic 6.0 environment.

The algorithm consisted of background segmentation, raisin selection and feature extraction. The developed algorithm initially extracts the raisins by removing the background from the taken images. It then sorts the raisins according to their Hue, Saturation and Intensity (HSI) color features. By a suitable combination of length and HSI color values raisins were graded it two classes. The final step in the algorithm was the calculation of the center of gravity of each raisin to be later used for automatic sorting and rejection of bad raisins. In order to evaluate the precision of the sorter statistical analysis was carried out. Experimental results indicated the accuracy of the proposed system is about $93 \%$.

\section{CONCLUSION}

A faster, automatic, precise systems for the sorting cleaning of rice grains and is designed. The above systems can replace the mechanical, conventional methods. These above systems are automatic. Speed of the separation process is very high. Accuracy of the process is very high.

\section{REFERENCES}

[1] Kavitha tated, Sunil Morade, "Application of Image processing for automated cleaning of Rice", 1st International Conference on Recent Trends in Engineering and Technology, March-2012.

[2] Bharat K, Patell R, C. Patel, "An Efficient Method for Detecting Impurity from Rice Mixture Using Digital Embedded System", IJSRD - International Journal for Scientific Research \& Development| Vol. 1, Issue 5, 2013 | ISSN (online): 2321-0613.

[3] Smita Shelke, Prof. A.P Phatale, "An automatic Grading system based on machine vision", International Journal of Innovative Research in Electrical, Electronics, Instrumentation and Control Engineering, vol.1, Issue 4,july 2013.

[4] Harpreet Kaur, Baljit Singh, "Classification and Grading of Rice using multi class SVM", International Journal of Scientific and Research publications, vol.3, Issue 4, April-2013.

[5] Chetna V. Maheshwari, " Machine vision Technology for ORYZA SATIVA L.(RICE)”, International Journal of Advanced Research in Electrical, Electronics and Instrumentation Engineering, Vol.2, Issue 7, July2013.

[6] Ms.Shubhada.K, Nagrale,Mr.S.T.Bagde, "Application of Image Processing for development of automated Inspection system", International Journal of Computational Engineering Research, vol.3, Issue 3, 2013.

[7] Chetna V. Maheshwari, "non-destructive quality analysis of indian gujarat-17 oryza sativa ssp indica(rice) using image processing", International Journal of Computer Engineering Science (IJCES) Volume 2 Issue 3 (March 2012) ISSN : 2250:3439.

[8] Latha, J. and N. Devarajan, "In process vision inspection system for sorting using image processing technique", ISSN 1549-3636 Journal of Computer Science 8 (4): 528-532, 2012.

[9] Roya Hassankhani, Hossein Navid, et al, "Potato Sorting in Machine Vision System: Based on Color, Size and Defect Detection", Lambert Publisher, 2012.

[10] Manish Chhabra, Rohan Gaur and Parminder Singh Reel, "Automated detection of fully and Partially Roped Mango by Machine Vision", International Conference in Recent Trends in Information Technology and Computer Science, pp.26-31, 2011.

[11] Mahdi Abbasgolipour, "Sorting Raisins by machine vision system", Moderrn Applied Science, vol.4, February 2010 